\title{
Genistein downregulates presenilin 1 and ubiquilin 1 expression
}

\author{
NAOKO OKUMURA*, HITOMI YOSHIDA*, YURI NISHIMURA, \\ MUTSUMI MURAKAMI, YASUKO KITAGISHI and SATORU MATSUDA
}

Department of Environmental Health Science, Nara Women's University, Nara 630-8506, Japan

Received August 5, 2011; Accepted October 25, 2011

DOI: $10.3892 / \mathrm{mmr} .2011 .648$

\begin{abstract}
The aim of this study was to determine the effects of several food ingredients and chemical inhibitors on the expression of presenilin, a molecule involved in $\gamma$-secretase activity and the generation of amyloid- $\beta$ peptide in Alzheimer's disease. Western blotting revealed the downregulation of presenilin 1 protein expression by stimulation with genistein in vitro, while the effects on presenilin 1 gene expression examined by reverse transcriptase-polymerase chain reaction (RT-PCR) were unaltered in Daudi cells. Genistein likely downregulates presenilin via the inhibition of ubiquilin 1 expression in lymphoid cells. Our findings provide new insights that may help to establish preventive strategies against Alzheimer's disease.
\end{abstract}

\section{Introduction}

Alzheimer's disease (AD) is a neurodegenerative disease with no cure and is the most common cause of dementia. Excessive accumulation of amyloid- $\beta$ seems to be the primary pathological event leading to AD (1). Neurotoxic amyloid- $\beta$ is a peptide generated via cleavage of amyloid- $\beta$ precursor protein (APP) by the $\gamma$-secretase complex. Presenilins are catalytic members of the complex, and mutations in the presenilin gene are the major cause of familial AD (2). Presenilin is involved in several biological functions, but is well known for its role in the generation of the amyloid- $\beta$ peptide in AD. It is therefore considered to be an important therapy target against the $\mathrm{AD}$ disorder.

As the Indian diet is rich in certain spices, the prevalence of AD patients in India is considerably low (3). However, the precise molecular mechanism for the effect of these spices is largely undefined, and limited data and evidence have been provided at the molecular level (4). Recent in vivo studies have indicated that curcumin is able to reduce amyloid- $\beta$-related pathology in transgenic AD mouse models via unknown

Correspondence to: Dr Satoru Matsuda, Department of Environmental Health Science, Nara Women's University, Kita-Uoya Nishimachi, Nara 630-8506, Japan

E-mail:smatsuda@cc.nara-wu.ac.jp

*Contributed equally

Key words: presenilin 1, ubiquilin 1, genistein, Alzheimer's disease, downregulation molecular mechanisms (5). Curcumin is a small fluorescent compound in the widely used culinary spice, turmeric, which possesses potent biological activities, including anti-inflammatory, anti-fibrilogenic activities, antioxidant activities, chemo-preventative effects, and effects on protein trafficking (6). It is also reported that curcumin lowers amyloid- $\beta$ protein levels by attenuating the maturation of APP in the secretory pathway (5). Curcumin led to a significant reversal of structural changes in dystrophic dendrites including abnormal curvature and dystrophy size (7). We also showed that curcumin induced the downregulation of the presenilin 1 protein in Jurkat cells (8). These data suggest that curcumin reverses the AD pathology and provides a mechanism of action for the attenuattion of amyloid- $\beta$ pathology by curcumin.

A better understanding of presenilin might help to improve the development of drugs for AD and for other neurological diseases (9). Therefore, high priority should be given to basic research and development aimed at elucidating the mechanism of action underlying presenilin expression. As ubiquilin expression modulates biogenesis and endoproteolysis of presenilin (10), we evaluated the biochemical effects of several food ingredients and chemical inhibitors on presenilin and ubiquilin expression in cultured human cells.

\section{Materials and methods}

Cell culture. The human cell lines, Daudi, Jurkat, U937 and K562, were maintained in RPMI-1640 supplemented with $10 \%$ fetal bovine serum (FBS), penicillin and streptomycin at $37^{\circ} \mathrm{C}$ in a humidified atmosphere containing $5 \% \mathrm{CO}_{2}$.

Preparation of reagents. The reagents were dissolved in ethanol and subsequently diluted to a stock concentration of $10 \mathrm{mM}$ and stored at $-20^{\circ} \mathrm{C}$ until use. For the cell treatments, a range of 0.5-10.0 $\mu \mathrm{l}$ was added to $1 \mathrm{ml}$ of the cell culture medium.

Reverse transcriptase-polymerase chain reaction ( $R T-P C R)$. Ubiquilin 1, presenilin 1 and GAPDH mRNAs were analyzed by semi-quantitative RT-PCR. Total RNA was extracted by an RNA isolation kit (Takara, Japan). Two micrograms of total RNA was reverse-transcribed using the Phusion RT-PCR kit (NEB) as described in the manufacturer's protocol. Cycle-based PCR was used to semi-quantitate the ubiquilin 1 and presenilin 1 gene levels. GADPH was also used as an internal loading control. All samples were analyzed within 3 months after collection. The primers used 
$\begin{array}{llllllll}1 & 2 & 3 & 4 & 5 & 6 & 7 & 8\end{array}$

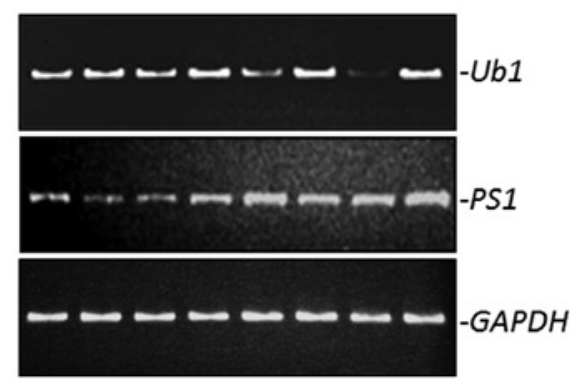

Figure 1. Genistein reduces the expression of ubiquilin 1 in Daudi cells. Semi-quantitative RT-PCR was performed using primers specific to presenilin 1, ubiquilin 1 or GAPDH in 100 ng total RNA prepared from Daudi

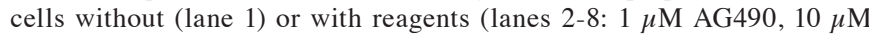
MG132, $20 \mu \mathrm{M} \beta$-secretase inhibitor II, $20 \mu \mathrm{M}$ lactacystin, $10 \mu \mathrm{M}$ ALLN, $50 \mu \mathrm{M}$ genistein, and $50 \mu \mathrm{M}$ daidzein, respectively) for $24 \mathrm{~h}$. Specific expression was determined in relation to the expression of the housekeeping gene GAPDH used as an internal loading control. At least four independent experiments were carried out, and typical paired results are documented.

for the PCR were designed as follows: ubiquilin 1 forward, TGCTGCAGGCTCTTGCTGGA; ubiquilin 1 reverse, TGGCTGGGAGCCCAGTAACCT, (expected size 179 bp); presenilin 1 forward, GGTCCACTTCGTATGCTGGT; presenilin 1 reverse, GCTGTTGCTGAGGCTTTACC, (expected size 404 bp); GAPDH forward, TCCCATCACCATCTTCCA; GAPDH reverse, CATCACGCCACAGTTTCC, (expected size 376 bp). For real-time PCR, the reactions were performed using a real-time PCR system (Illumina Inc., San Diego, CA, USA) using KAPA SYBR FAST reaction mix (Genetics, Japan). Thermo-cycling was carried out according to the manufacturer's instructions at a $60^{\circ} \mathrm{C}$ annealing temperature in a final volume of $10 \mu \mathrm{l}$ including Taq DNA polymerase.

Western blot analysis. Equal amounts of protein samples were used for Western blot analysis using anti-ubiquilin 1 (GeneTex Inc., Irvine, CA, USA), anti-presenilin 1 (GenScript USA Inc., Piscataway, NJ, USA), anti-presenilin 2 (GenScript) and anti-Erk2 (Epitomics Inc., Burlingame, CA, USA) antibodies, and quantified by densitometry. All Western blots were repeated at least three times and representative data are shown.

\section{Results and discussion}

Several reagents involved in cell signaling and/or inhibition of the proteasome system were added to the cell culture medium of Daudi, Jurkat, U937 or K562 cells, and the levels of the genes, including ubiquilin 1 and presenilin 1, were examined. We employed RT-PCR analysis to quantify the expression level of the genes. Total RNA was isolated $24 \mathrm{~h}$ after treatment, and the levels of mRNA were determined by conventional semi-quantitative RT-PCR. As shown in Fig. 1, expression levels of presenilin 1, presenilin 2 (data not shown) and housekeeping gene GAPDH were unaltered throughout the treatments of the indicated compounds compared with the ethanol vehicle, whereas the expression level of the ubiquilin 1 gene was markedly decreased upon treatment of genistein. Similar results were also obtained from the quantitative real-

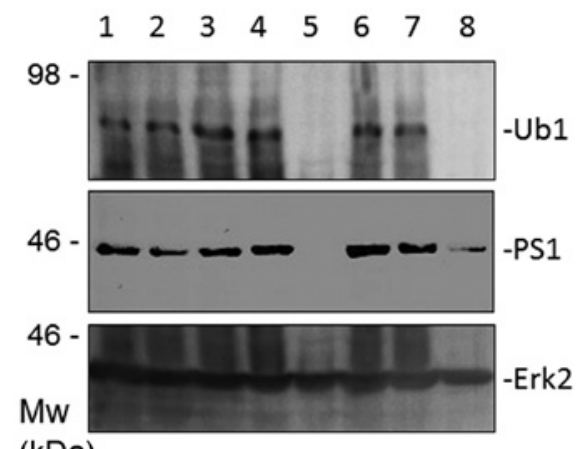

$(\mathrm{kDa})$

Figure 2. Genistein and AG490 downregulate expression of ubiquilin 1 and presenilin protein in Daudi cells. Daudi cells were treated without (lane 1) or with reagents (lanes 2-8: $1 \mu \mathrm{M}$ dexamethasone, $0.2 \mu \mathrm{M} 17-\beta$-estradiol, $10 \mu \mathrm{g} / \mathrm{ml}$ mixed food isoflavones, $50 \mu \mathrm{M}$ genistein, $50 \mu \mathrm{M}$ daidzein, $1 \mu \mathrm{M}$ AG490, and $50 \mu \mathrm{M}$ AG490, respectively) for $48 \mathrm{~h}$. After treatment, cell lysates were isolated, and Western blotting was performed using antibodies specific to presenilin 1, ubiquilin 1 and Erk2. At least three independent experiments were carried out, and typical paired results are shown.
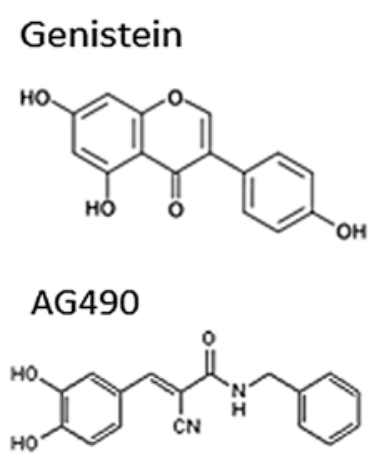

Figure 3. Chemical structure of genistein and AG490.

time PCR analysis (data not shown). There was also little difference among the results of the gene expressional profile for the Daudi, Jurkat, U937 and K562 cells (data not shown). To exclude the possibility of carry-over DNA contamination, reactions containing all RT-PCR reagents, including primers without sample RNA, were preformed as negative controls. No such contamination was detected (data not shown).

To further examine the expression status of the protein level, Western blot analysis was performed to analyze the ubiquilin 1 and presenilin 1 protein in the cells stimulated by the reagents. As shown in Fig. 2, 50 $\mu \mathrm{M}$ of genistein and the tyrosine kinase inhibitor (AG490) markedly reduced both ubiquilin 1 and presenilin 1 protein expression, when cell cultures had been treated with the reagents for $48 \mathrm{~h}$. Note that AG490 reduced presenilin 1 protein expression, whereas its mRNA expression was unchanged (Fig. 1). Equal amounts of protein resources from the cell lysates were confirmed by immunoblotting for Erk2. Chemical molecular structures of genistein and AG490 are shown in Fig. 3. As genistein displays activities both in the inhibition of several tyrosine kinases and in activation of estrogen receptor $\beta$, we then examined whether AG490 and estradiol synergistically reduce presenilin 1 expression. After treating the cells with different concentrations of estradiol and AG490, we found 


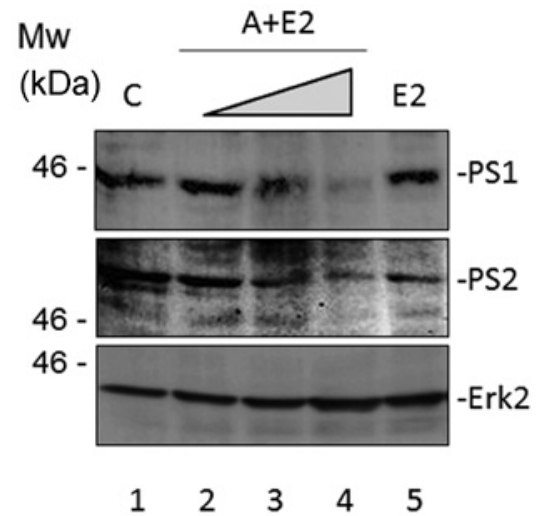

Figure 4. Dose-dependent inhibition of protein expression of presenilin 1 and presenilin 2 by AG490 and estradiol. Daudi cells were treated without (lane 1) or with $1 \mu \mathrm{M}$ AG490 plus $1 \mathrm{nM}$ (lane 2), $10 \mathrm{nM}$ (lane 3) and $100 \mathrm{nM}$ (lane 4) of 17- $\beta$-estradiol (A+E2) or $0.2 \mu \mathrm{M} 17-\beta$-estradiol alone (lane 5) for $48 \mathrm{~h}$. The levels of protein were detected by Western blot analysis using antipresenilin 1 and anti-presenilin 2 antibodies, as described in Fig. 2. Western blot analysis with the anti-Erk2 antibody was also carried out to ensure equal levels of protein loading.

that the protein expression of presenilin 1 and 2, but not Erk2, was decreased with increasing concentrations of estradiol with AG490. A final concentration of $100 \mathrm{nM}$ of estradiol and $1 \mu \mathrm{M}$ AG490 inhibited presenilin 1 expression by more than 95\% in Jurkat cells (Fig. 4). These data showed that AG490 and estradiol had a combined effect on the downregulation of presenilin 1 and 2. AG490 and estradiol also downregulated presenilin 1 in a dose-dependent manner in K562 cells (data not shown). Presenilin expression may be regulated by the estrogen receptor and/or tyrosine kinase signaling. Ubiquilin has been originally identified as a presenilin-interacting protein and has been shown to stabilize presenilin (11). It has also been shown that ubiquilin is associated with AD $(12,13)$. Accordingly, our results also suggest that genistein may downregulate presenilins probably via the inhibition of ubiquilin 1 expression in lymphoid cells.

Although the molecular mechanisms by which ubiquilin 1 is regulated have not yet been identified, our findings provide new insight and may help to establish preventive strategies against AD. Soy food is high in genistein, which can be safely orally administered to humans; however, some suspicions conerning its effectiveness in clinics may arise for fear of the expected assimilated-concentration in vivo (14). Considering the presence of the blood-brain barrier, it is reasonable to conclude that the genistein concentration in the brain may be lower than the effective concentration. As for prion disease, the lympho-reticular system has been implicated as the route of transmission of pathogenic prion from the gut to the brain. Indeed, T cells are considered to be important effector cells contributing to neuronal damage in degenerative CNS disorders (15). This implies that some lymphocytes can interact with neuronal cells in the brain. It is, therefore, likely that there is a lymphocytic cell-bridge between the gut lumen, where genistein concentrations could be more than $1 \mathrm{mM}$, and brain neurons on which APP must be the target for presenilins of lymphocytes. In the present study, we showed that genistein and another tyrosine kinase inhibitor AG490 downregulate the presenilin protein, which plays a crucial role in $\gamma$-secretase activity, in a dose-dependent manner. Recently, it has been reported that ubiquilin 1 regulates proteasomal degradation of proteins including presenilin (16). Various herbs and spices including genistein or curcumin may regulate this degradation. Further studies including in vivo experiments are required to investigate the effects of genistein on proteins implicated in Alzheimer's disease.

\section{Acknowledgements}

This study was supported by grants-in-aid from the Ministry of Education, Culture, Sports, Science and Technology of Japan and Nara Women's University Intramural Grant for Project Research. In addition, this work was supported in part by a grant from the Fuji Foundation for Protein Research.

\section{References}

1. Bekris LM, Yu CE, Bird TD and Tsuang DW: Genetics of Alzheimer disease. J Geriatr Psychiatry Neurol 23: 213-227, 2010.

2. Quiroz YT, Ally BA, Celone K, McKeever J, Ruiz-Rizzo AL, Lopera F, Stern CE and Budson AE: Event-related potential markers of brain changes in preclinical familial Alzheimer disease. Neurology 77: 469-475, 2011.

3. Ganguli M, Chandra V, Kamboh MI, Johnston JM, Dodge HH, Thelma BK, Juyal RC, Pandav R, Belle SH and DeKosky ST: Apolipoprotein E polymorphism and Alzheimer disease: The Indo-US Cross-National Dementia Study. Arch Neurol 57: 824-830, 2000.

4. Nishimura Y, Kitagishi Y, Yoshida H, Okumura N and Matsuda S: Ethanol extracts of black pepper or turmeric down-regulated SIRT1 protein expression in Daudi culture cells. Mol Med Rep 4: 727-730, 2011.

5. Zhang C, Browne A, Child D and Tanzi RE: Curcumin decreases amyloid- $\beta$ peptide levels by attenuating the maturation of amyloid- $\beta$ precursor protein. J Biol Chem 285: 28472-28480, 2010.

6. Yu YC, Miki H, Nakamura Y, Hanyuda A, Matsuzaki Y, Abe Y, Yasui M, Tanaka K, Hwang TC, Bompadre SG and Sohma Y: Curcumin and genistein additively potentiate G551D-CFTR J Cyst Fibros 10: 243-252, 2011.

7. Garcia-Alloza M, Borrelli LA, Rozkalne A, Hyman BT and Bacskai BJ: Curcumin labels amyloid pathology in vivo, disrupts existing plaques, and partially restores distorted neurites in an Alzheimer mouse model. J Neurochem 102: 1095-1104, 2007.

8. Yoshida H, Okumura N, Nishimura Y, Kitagishi Y and Matsuda S: Turmeric and curcumin suppress presenilin 1 protein expression in Jurkat cells. Exp Ther Med 2: 629-632, 2011.

9. Crews L, Rockenstein E and Masliah E: APP transgenic modeling of Alzheimer's disease: mechanisms of neurodegeneration and aberrant neurogenesis. Brain Struct Funct 214: 111-126, 2010.

10. Massey LK, Mah AL and Monteiro MJ: Ubiquilin regulates presenilin endoproteolysis and modulates gamma-secretase components, Pen-2 and nicastrin. Biochem J 391: 513-525, 2005.

11. Mah AL, Perry G, Smith MA and Monteiro MJ: Identification of ubiquilin, a novel presenilin interactor that increases presenilin protein accumulation. J Cell Biol 151: 847-862, 2000.

12. Bertram L, Hiltunen M, Parkinson M, et al: Family-based association between Alzheimer's disease and variants in UBQLN1. N Engl J Med 352: 884-894, 2005.

13. Li A, Xie Z, Dong Y, McKay KM, McKee ML and Tanzi RE: Isolation and characterization of the Drosophila ubiquilin ortholog dUbqln: in vivo interaction with early-onset Alzheimer disease genes. Hum Mol Genet 16: 2626-2639, 2007.

14. Mancuso C, Siciliano R and Barone E: Curcumin and Alzheimer disease: this marriage is not to be performed. J Biol Chem 286: le3, 2011.

15. Meuth SG, Herrmann AM, Simon OJ, et al: Cytotoxic CD8 ${ }^{+}$ $\mathrm{T}$ cell-neuron interactions: perforin-dependent electrical silencing precedes but is not causally linked to neuronal cell death. J Neurosci 29: 15397-15409, 2009.

16. Viswanathan J, Haapasalo A, Böttcher C, et al: Alzheimer's disease-associated ubiquilin-1 regulates presenilin-1 accumulation and aggresome formation. Traffic 12: 330-348, 2011. 